\title{
INVESTIGATION OF COCOA HUSKS DENSIFICATION POSSIBILITIES
}

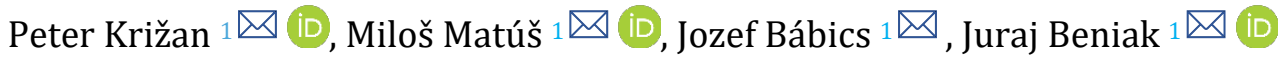 \\ ${ }^{1}$ Institute of Manufacturing Systems, Environmental Technology and Quality Management, Faculty of \\ Mechanical Engineering, Slovak University of Technology in Bratislava, Slovak Republic Bratislava. Slovakia
}

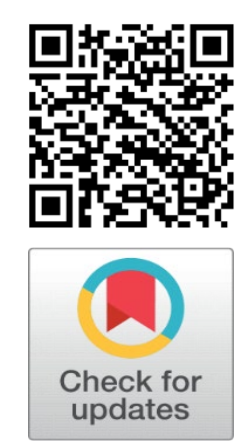

Received 6 December2021

Accepted 16 December 2021

Published 31 December 2021

\section{CorrespondingAuthor}

Peter Križan, peter.krizan@stuba.sk

DOI

10.29121/granthaalayah.v9.i12.2021 4446

Funding: This research received no specific grant from any funding agency in the public, commercial, or not-for-profit sectors.

Copyright: (C) 2021 The Author(s). This is an open access article distributed under the terms of the Creative Commons Attribution License, which permits unrestricted use, distribution, and reproduction in any medium, provided the original author and source are credited.

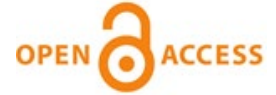

\section{ABSTRACT}

There are many technological and material variables that can be identified during biomass densification. These factors have a significant impact on the final quality of the biofuels (pellets, pellets, briquettes). This paper will discuss the research results regarding the relationships between technological variables and material variables in densification of cocoa husks. Cocoa husks are a common product of a food factory as an unutilized raw feedstock. This experimental research aims to investigate densification possibilities of cocoa husks and determine the effects of compression pressure and particles size on final biofuels quality. The single-axis experiment described in this paper involved experimental pressing stands. It was possible to determine the effect of these variables on final pellets density. These variables have mutual interactions that can affect final pellets quality, which is why they are important during densification. Experimental research at semi-production pelleting plants also proved the impact of raw particles size on final biofuels. These research findings concern pelleting and briquetting options.

Keywords: Biomass, Cocoa Husks, Densification, Extrusion Density, Compression Pressure, Particle Size

\section{INTRODUCTION}

Densification of biomass is a mass- and energy recovery process that uses low bulk densities for raw materials. The end-user's expectations regarding the heating system and handling properties will determine the quality of the final product of densification Fernandes and Costa (2012), Garcia Maraver et al. (2014). The quality of biofuels depends on the characteristics of the raw material (feedstock), which includes its biomass type, moisture content, and particle size. Quality management of the manufacturing process, including operating conditions, technological variables and pelletizer type, as well as the binding agent, will also affect the quality of the final product Kracík et al. (2016), Arce et al. (2013). In order to cause problems in combustion systems, low-quality pellets may have undesirable effects such as fouling, corrosion or slugging. They can also produce large amounts of particulate and gaseous matter (PM) emissions Fernandes and Costa (2012), Garcia Maraver et al. (2014), Arce et al. (2013). Many projects and applications exist that allow energy recovery from different raw materials. The development of biomass energy recovery technology and the gradual decrease in the amount of wood biomass usable, has increased the need for processing other types of biomasses, agricultural - herbaceous biomass Križan (2015) . Other hands, some agricultural residues, where the energy potential is not. proper, can be very easily used for livestock husbandry purposes. 
In these cases, densification process is also very helpful Mostly due to negative Mostly due to negative ratio weight/volume, which is increasing the transport costs, but also due to requirements for livestock feed or bedding.

Cocoa husks as an unused raw feedstock after chocolate production belongs to the group of herbaceous biomasses. Cocoa is not typical Slovakian plant. But in Slovakia is wide chocolate production, where the husks remaining as unused source of waste. This experimental research aims to investigate the effects of technological variables on final biofuels quality. Every type of raw feedstock is unique in its chemical composition, properties and behavior during mechanical processing and densification. A small change in the properties can have a significant impact on the final quality of biofuels Križan (2015). Different raw feedstock properties require different approaches and technological conditions Križan et al. (2015). It is essential to determine and establish the optimal parameters of densification for each type of raw material in order to design an economically efficient process of biomass treatment Križan et al. (2015), Križan (2021). The applicable technical standards regarding the quality of biofuels must be adhered to. The EN Standards Standard EN ISO 17225-2: (2014), Standard EN ISO 17225-6:(2014) provide a basis for evaluating the quality of biofuels using mechanical indicators (bulk density and mechanical durability, etc.) both from a point of view and as thermal-chemical indicators points of view.

The assignment was based on industry cooperation. Its goal was to efficiently process the company's technological scrap - cocoa husks - into a form that is more suitable for long-distance transportation and perhaps use as bedding for livestock. Expert analysis was performed to confirm the use of densification technology for the treatment of cocoa husks. It also sought to identify the technological parameters that influence the quality of extrusions. Simple analyses were performed on the cocoa husk samples that were not crushed. There are important differences between the wood biomass and cocoa husks in terms of densification. Some problems arise from the raw material behavior, properties and composition, as well as from the structure of the raw material. These parameters can also influence the final biofuels made from cocoa husks Kracík et al. (2016). It was essential to assess the impact of technological variables and raw materials treatment variables on final biofuels' quality as well as densification options for extrusions manufacturing. This was the main objective. These experimental results are important from a biofuel production and densification machine constructions perspective Križan (2015). This task is solved by the need for practice. The company must improve the quality of its pellets.

\section{MATERIALS AND METHODS}

Based on our experience and knowledge from measurements, we can determine the impact of technological variables (compression pressure) as well as the effect of raw material parameters (type of feedstock, moisture content, and particle size) upon biofuels quality Križan (2015), Križan et al. (2015). This experimental study focused on the effects of compression pressure and particle distribution on final biofuels quality. These variables influence the conditions of the pressing chamber during densification, which in turn influences final biofuels quality as well as the stabilization time. The biofuels stabilization interval is the time during which dilatation takes place Standard DIN $52182:(1976)$. It is also the time period during which the biofuel stabilizes. The biofuel stabilization process takes around 24 hours depending on the type of raw feedstock used and the densification technology employed. However, it can take even longer. The standard DIN 52182 
Standard DIN 51731 :(1996) describes how to detect biofuel density after stabilization. Biofuel dilatation refers to a process in which biofuel dimensions or weight change (diameter and length, respectively). These changes are caused by the raw feedstock properties as well as external variables in the densification process. The biofuel quality is directly affected by dilatation Standard DIN 52182 :(1976). The combination of all the variables and parameters mentioned can have a significant impact on densification, especially from the quality perspective of final biofuels Križan (2015). We can determine the best conditions for each input type (type of raw material and its parameters).

In this study the cocoa husks were used. Initial bulk density of used cocoa husks according to EN IS017828 Standard EN ISO 17828 :(2015) was measured. Properties of supplied raw material are in following Table 1.

\begin{tabular}{|ccc|}
\hline Table 1 Samples of the supplied and treated raw material \\
\hline Observed parameter & $\begin{array}{c}\text { Coarse fraction } \\
\text { (Supplied sample) }\end{array}$ & $\begin{array}{c}\text { Fine fraction } \\
\text { (Shredded sample) }\end{array}$ \\
\hline Moisture content $(\%)$ & 5.723 & 5.428 \\
\hline Bulk density $\left(\mathrm{kg} / \mathrm{dm}^{3}\right)$ & 0.15055 & 0.44363 \\
\hline Combustion heat $(\mathrm{MJ} / \mathrm{kg})$ & 16.5912 & - \\
\hline
\end{tabular}

Before laboratory testing and trials, the sample was refined. Crushing was used to produce two samples with different fractional compositions. The client supplied the first material sample to be tested and pelletized. It was the untreated fraction (coarse fraction). The second sample was crushed (fine fraction). The Stoza SV5 hammer mill, with a $4 \mathrm{~mm}$ sieve, was used to adjust the percentage of cocoa husks. Due to the nature and size of the cocoa husks, the provided fraction was sufficient for the pelleting experiments. However, the pellets were only $20 \mathrm{~mm}$ in diameter. To determine the impact of the fraction composition on the final quality, crushing was done. Figure 1 shows the sample before crushing - the coarse fraction (supplied specimen) and Figure 2 after crushing (fine fraction).

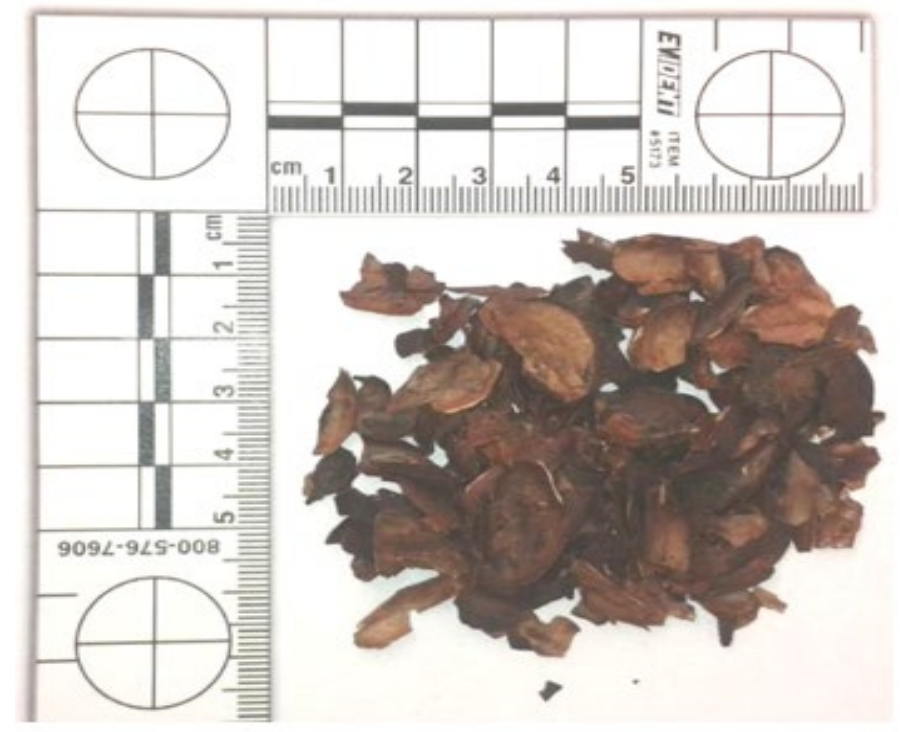

Figure 1 Cocoa husks - supplied (course fraction) 


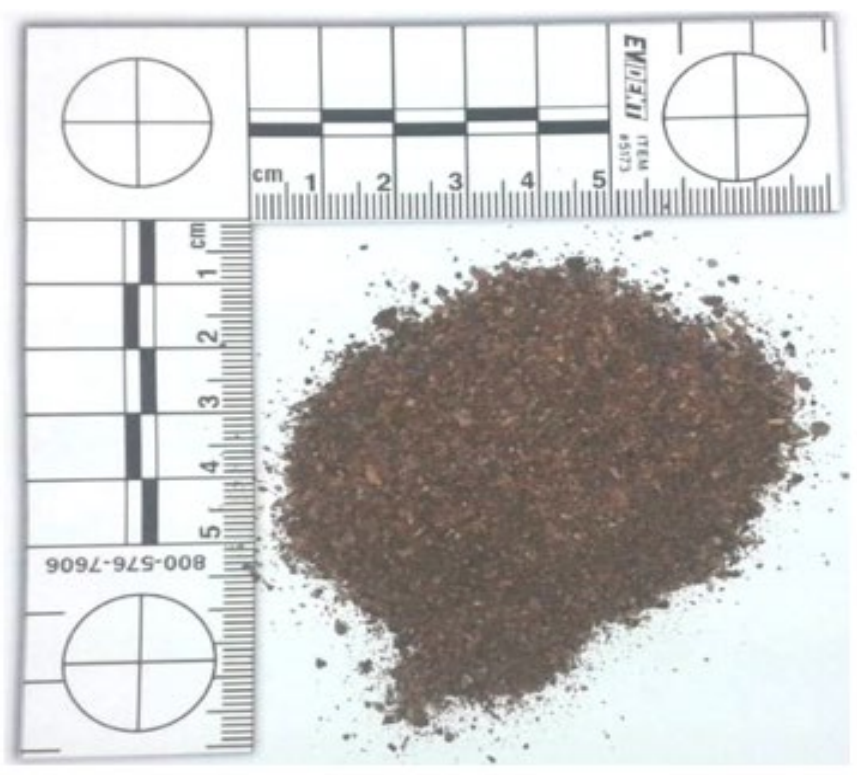

Figure 2 Cocoa husks - crushed (fine fraction)

The particle size distribution was then determined using particle size analysis with a Retsch AS 200 vibrating screening. Figure 3 shows the results. The fractions that contain a greater proportion of finer fractions can theoretically be more effective in terms of quality and densification technology. This can greatly improve the quality of extrusions.

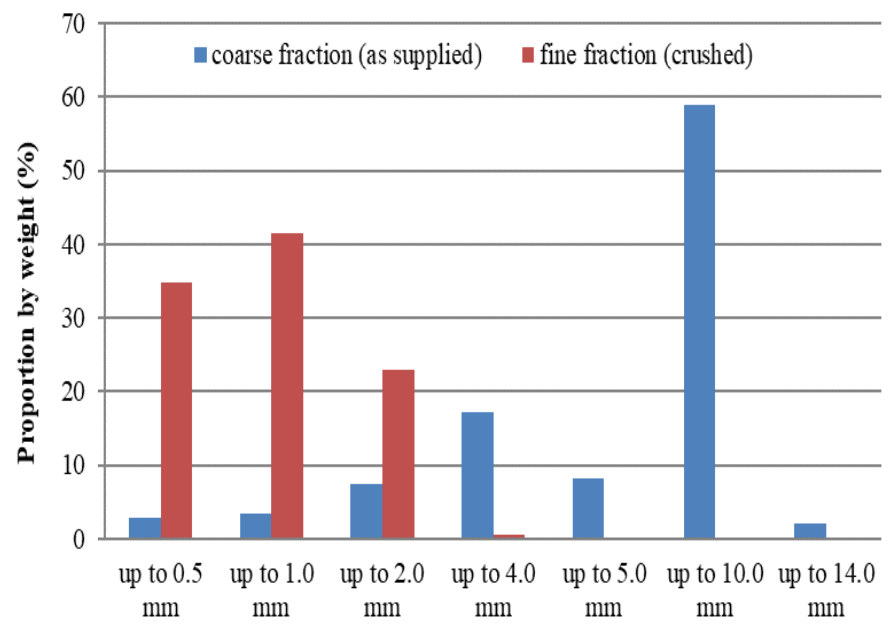

Figure 3 Granulometric analysis and proportion of individual fractions throughout the volume

A Kern MRS 120-3 balance was used to measure the moisture content of cocoa husks prior to disintegration and pelletization. The raw feedstock was heated (gravimetric method for moisture content measurement) Standard EN ISO 18134-3 :(2016) until it reached a constant weight. This study used only one level of moisture content due to the complicated process of moisture content changes. Cocoa husks of the moisture content listed in Table 1 were used for disintegration, pelletizing, and another experimental research. The above-mentioned figures, as well as the results from the particle size analysis, show that crushing cocoa husks resulted in a 
significant increase in the volume of each individual fraction. The material sample parameters also changed (Table 1). Although the moisture content of the sample decreased slightly after crushing, it was not significant. The bulk density was a more important variable. The bulk density of the finer (crushed) sample was 2.94 times higher than that of the supplied sample. It can be said that crushing the material before transport can greatly reduce costs.

It was important to separate the experimental research into two phases in order to achieve the main goal:

1) Experimental research using an experimental pressing stand (Figure 4)

2) Semi-operation tests using production technological plant (Figure 5)

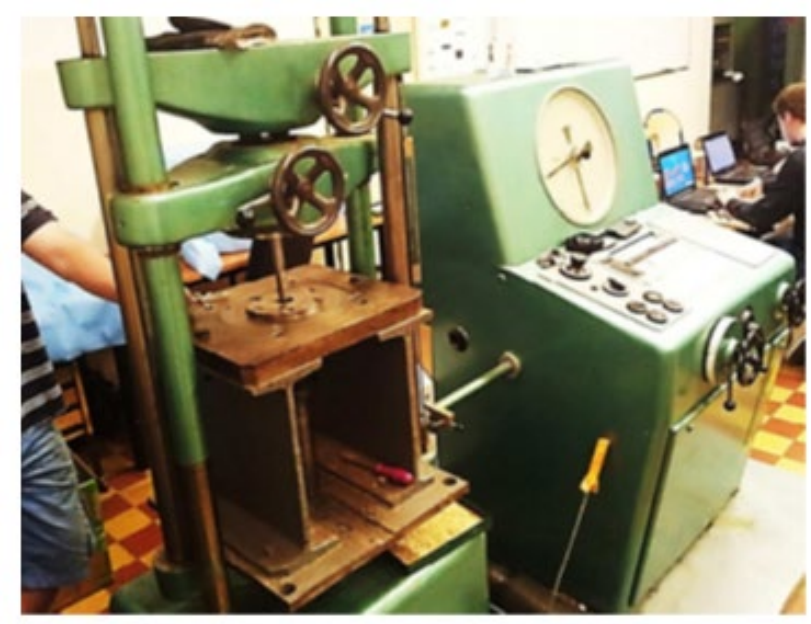

Figure 4 Hydraulic press with experimental pressing stand

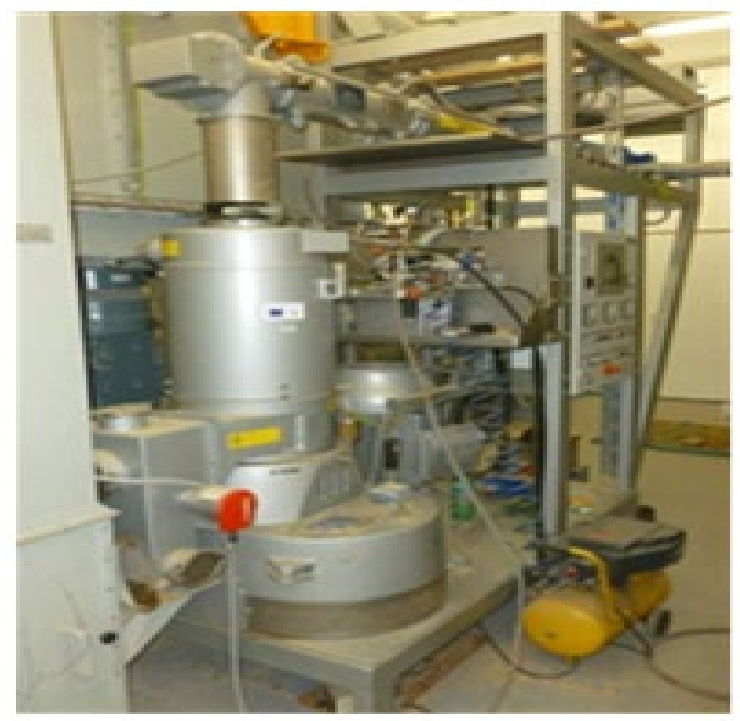

Figure 5 Pelletizing press KAHL 33-390

Experimental research was conducted in laboratory conditions at $23^{\circ} \mathrm{C}$. The raw feedstock matter did not permit for additional pressing temperatures Križan (2021). The primary goal of the first phase was to examine the effects of compression pressure and particle distribution on final biofuels quality, and biofuels dilatation. These results were achieved without the influence of extra 
pressing temperature Kaliyan and Morey Vance (2009), Nielsen et al. (2009). The experimental pressing stand used for the experiment was shown in Figure 4), which a vertical single-axis pressing is. The hydraulic press is equipped with a press stand that can be used to generate compression pressure at various levels. Experimental research was conducted according to the experimental plan. The range of compression pressure from 63 to $222 \mathrm{MPa}$ was tested. The raw material was introduced into the pressing chamber. Compression was then initiated using a hydraulic piston. The pressing stand was set up with a $140 \mathrm{~mm}$ long pressing chamber and a $20 \mathrm{~mm}$ diameter pressing stand. This was to achieve the research goal. For each setting, seven extrusions of a diameter of $20 \mathrm{~mm}$ were required to maintain the experimental plan. This number is the minimum value that can be processed using mathematical and statistical methods Križan (2015). According to previous experience, the holding time in the pressing chamber was selected. It's the 7 second compression pressure applied to each extrusion. There are many quality indicators that can be used to determine the quality of extrusions. In this instance, we used the extrusion density as an evaluation parameter. The extrusion's volume/weight ratio were used to calculate the extrusion density. Each extrusion was measured for its weight, length, and diameter. The extrusions' length and diameter were measured according to EN ISO 17829 Standard EN ISO 17829 :(2016). After that, the volume was calculated. The average density was then calculated for each setting. Once the extrusions have been stabilized, the extrusions' length, diameter and weight was measured again. This allows you to identify the dilatation of extrusions, and the impact of technological parameters on extrusions dilatation Standard DIN $52182:(1976)$.

\section{RESULTS AND DISCUSSIONS}

The figures below show graphically all the results from this investigation. They illustrate the interrelationship between different variables. These experimental results can be applied to choosing densification machine dimensions. They also provide a complete overview of the behavior and interactions of variables during the densification of cocoa husks. Next was laboratory testing of cocoa husks to determine their "pressability". This is the next phase, and it is necessary before compaction tests. The basic purpose of this testing is to confirm or deny the use of the cocoa husk processing technology. Laboratory testing can also be used to test the technology parameters and their effect on extrusion quality and dilation. Laboratory testing was done to confirm the suitability and quality of cocoa husk processing technology and to assess the effect of technological changes on extrusion quality and dilatation. Figure 6 and Figure 7 shows the extrusions of cocoa husks made from both fractions.

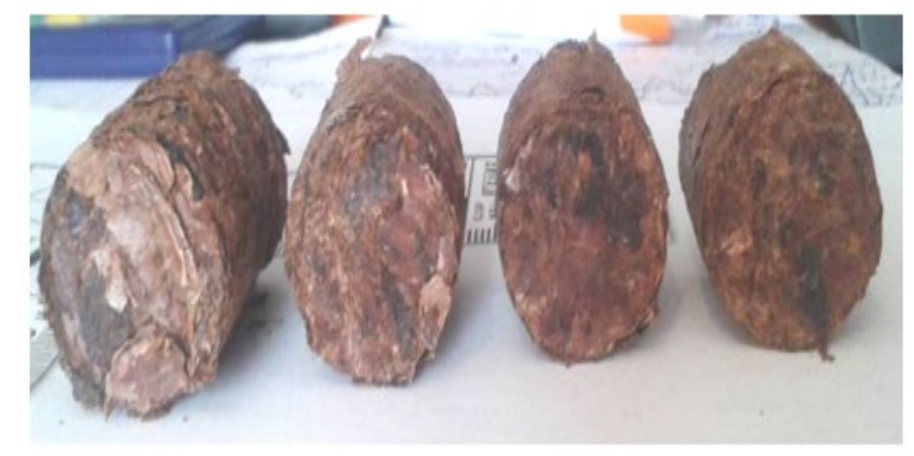

Figure 6 Extrusions of cocoa shells - coarse fraction 


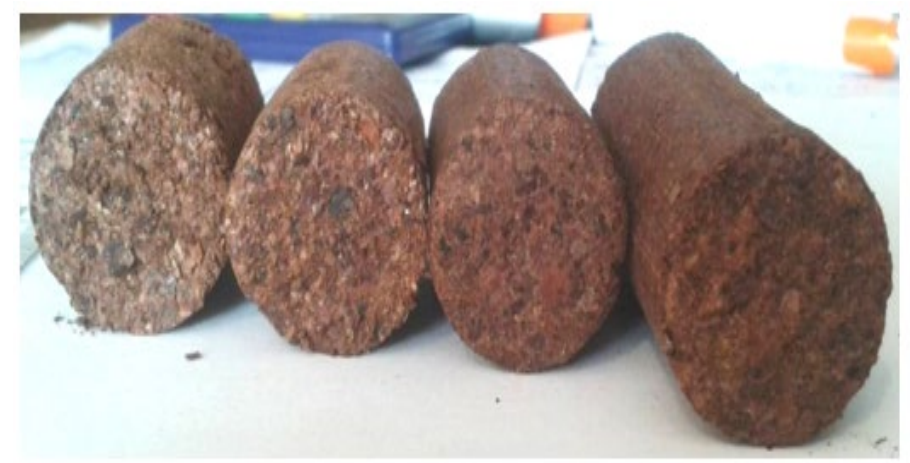

Figure 7 Extrusions of cocoa shells - fine fraction

A pressing experiment was conducted to test the effects of a change in compaction pressure. The pressure was set from 63 to $222 \mathrm{MPa}$. It was not necessary to experiment with the effects of changing the pressing temperature due to the nature and structure the input material. We have had good results pressing materials that are similar in chemical composition to the cocoa shells. Materials with low lignin or hemicellulose content, show that additional temperature has no significant effect on extrusions density during compaction Križan et al. (2015) It is not necessary to perform experiments outside of the specified intervals from a technical standpoint. After extrusion from the chamber, extrusions were weighed for their basic dimensions (diameter) and length. These are the graphical dependencies of the results.

The measured dimensions of the extrusion's length, diameter and weight were used to calculate density. The results were then indicated by graphical dependencies. The results on Figure 8 can be seen that dilatation also negatively affected the extrusions' size, which led to a decrease of density. After stabilization, the average density of extrusions dropped by $14.53 \%$. With Figure 9 it was confirmed again that increasing the compaction pressure results in a higher extrusion density. It was also observed that extrusion size is negatively affected by dilation, which results in a decrease of their density. The average density of compacts after stabilization fell by $7.66 \%$. This is lower than extrusions with finer input material.

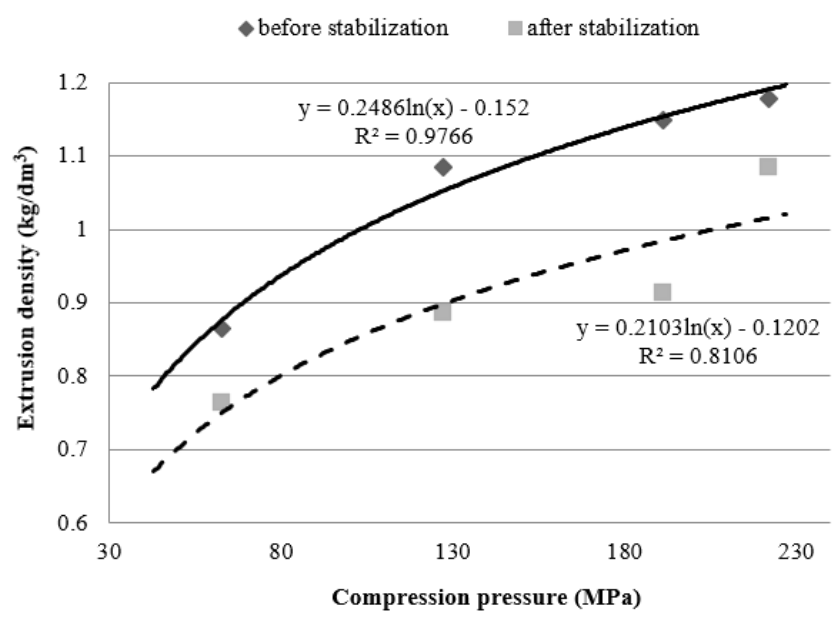

Figure 8 The extrusion density dependence on the compression pressure of input material (crushed fraction) 


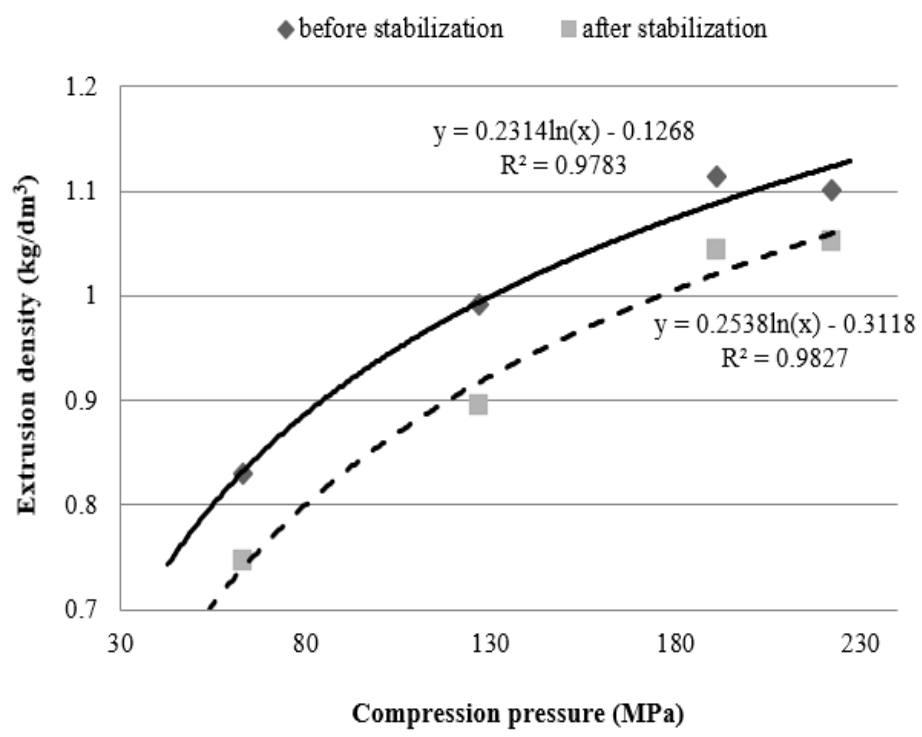

Figure 9 The extrusion density dependence on the compression pressure of input material (supplied fraction)

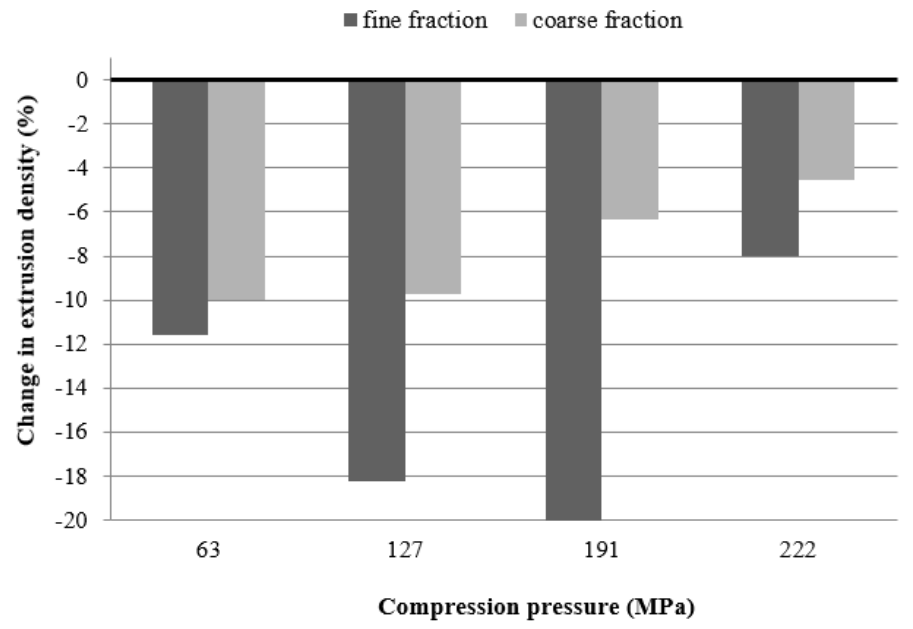

Figure 10 Dependence of the change in extrusion density on the compression pressure for both fraction sizes

Figure 10 clearly shows the change in density following stabilization. Both pressings of different fractions of cocoa husks result in a negative change. The dilation effect that caused the decrease in density was less noticeable for the uncrushed coarse fraction of cocoa husks. This trend is not evident in the results of pressing fine fraction (crushed cocoa husks). This is due to the differences in structure and representation of individual fractions within the pressed volume. This information is crucial when designing the pressing machine or dimensioning the tool. The results in Figure 11 show that the extrusion density values obtained from pressing for the fine and coarse fractions were different. However, the values obtained through pressing of the coarse fraction were superior. This can be explained by the stronger bonds formed between the material particles in the coarse fractions. The dependences in Figure 11 occur after stabilization of extrusions. In a 
condition that can be measured "in the field". The average extrusion densities achieved by pressing with coarse fractions were $1.99 \%$ higher than those obtained with fine fractions.

These results confirmed that cocoa husks are suitable for pressing technology. It was interesting to study the effects of compression pressure on the output material. This revealed that refinement (crushing), was not necessary. Tests revealed that coarse fractions (uncrushed), produced higher extrusion densities.

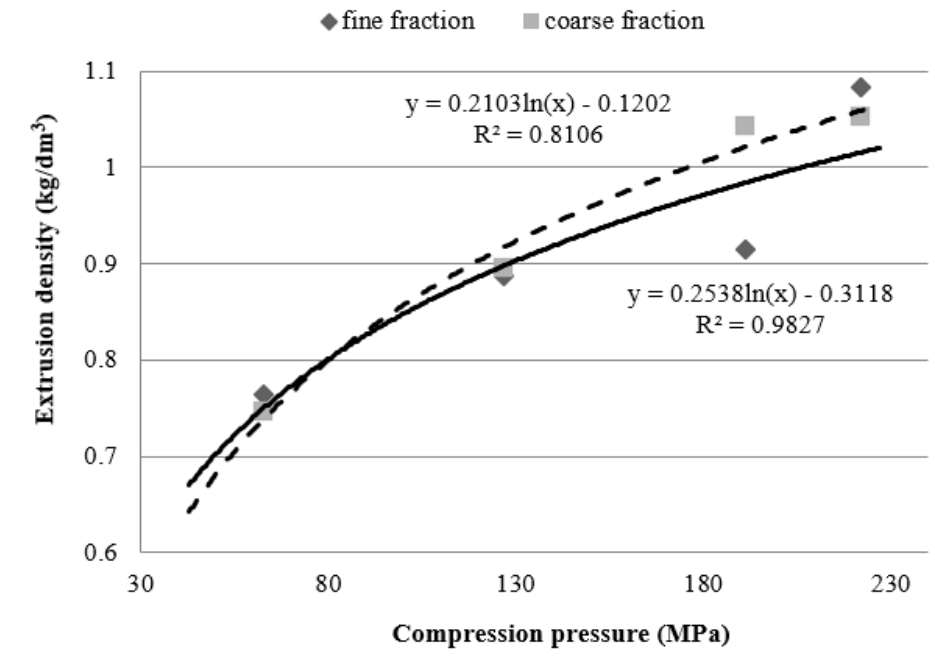

Figure 11 The extrusion density dependence on the compression pressure of input material (both fractions)

Based on the results, the clients decided to buy and operate the pressing technology within their production line. However, there was still the question of whether they would prefer briquetting or pelleting. Operational tests on cocoa husk-briquettes were conducted first to determine the best technology for the pressing. These tests were done on a BrikStar 200 hydraulic briquetting machine and a BL 50-350 mechanical crank press. There was a significant difference in the type and method of pressing. It was clear to see the difference in pressing type, hydraulic press vs. mechanical press. Despite all the possibilities and our efforts, we were unable to successfully test the hydraulic press. After being removed from the pressing chamber, the briquettes didn't fully compress and fall apart. This is due to both the properties of the briquetted materials and technological parameters. The optimal values were not possible due to the $55 \mathrm{~mm}$ size of the briquette. Figure 12 shows the remains of briquettes made from cocoa husks. These extrusions were not tested or verified.

A few extrusions were made on the mechanical crank press and then subjected to determination of specific weight - density of briquettes (Figure 13). The average value of specific weight achieved was $0.8516 \mathrm{~kg} / \mathrm{dm}^{3}$. The test was "successful" using the mechanical briquetting press, but it is possible to say that the resulting briquettes from cocoa husks in generally, were not enough compact. The results of briquetting tests have shown that optimization is required for the design parameters of a briquetting chamber, depending on the technology parameters of the press. This is the only way that you can improve the pressure conditions in the pressing chamber under these conditions. 


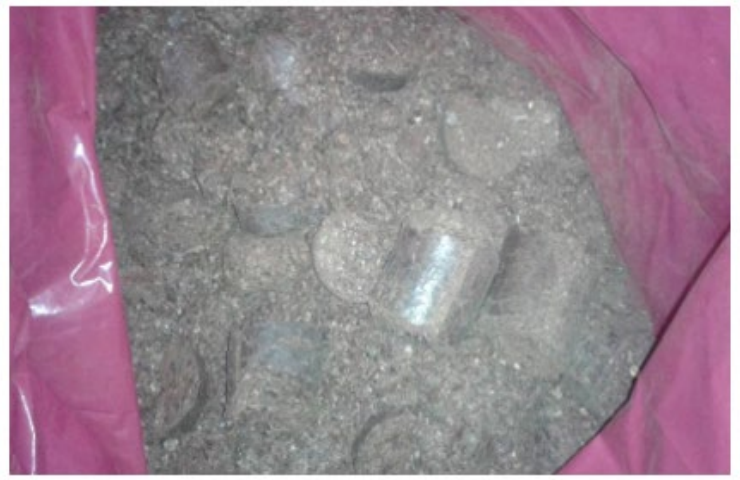

Figure 12 Failed extrusions of cocoa husks utilizing a hydraulic briquetting press

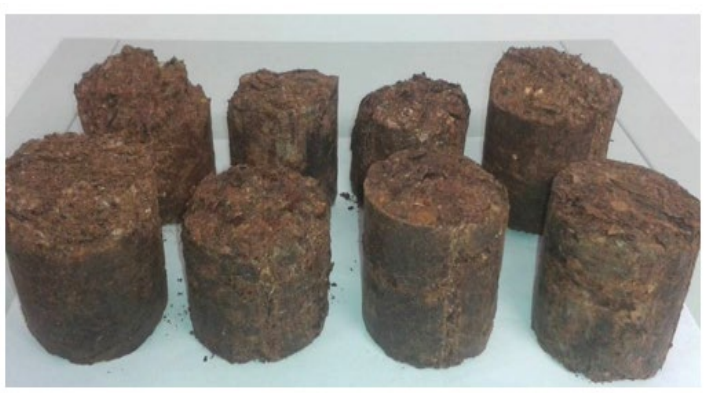

Figure 13 Briquettes made of cocoa husks produced using a mechanical briquetting press

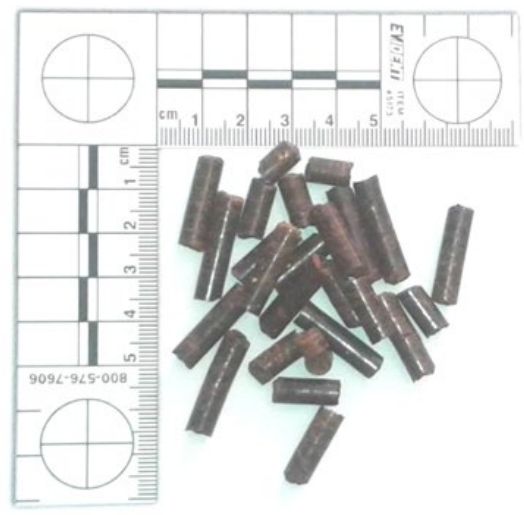

Figure 14 Cocoa husk pellets - course fraction

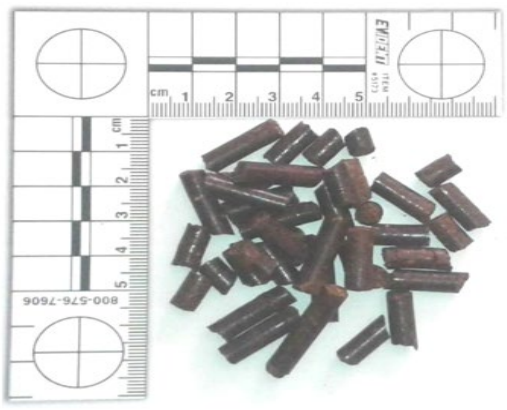

Figure 15 Cocoa husk pellets - fine fraction 


\begin{tabular}{|ccc|}
\hline \multicolumn{3}{|c|}{ Table 2 Properties of cocoa husks pellets } \\
\hline Observed parameter & Course fraction & Fine fraction \\
\hline Moisture content $(\%)$ & 7.609 & 7.276 \\
\hline Combustion heat $(\mathrm{MJ} / \mathrm{kg})$ & 18.5856 & 18.6141 \\
\hline Bulk density $\left(\mathrm{kg} / \mathrm{dm}^{3}\right)$ & 0.63654 & 0.63601 \\
\hline Particle density $\left(\mathrm{kg} / \mathrm{dm}^{3}\right)$ & 1.251 & 1.238 \\
\hline Mechanical durability (\%) & 68.99 & 70.58 \\
\hline Abrasion $(\%)$ & 38.32 & 46.15 \\
\hline Pellet hardness $(\mathrm{N})$ & 158 & 162.5 \\
\hline
\end{tabular}

The cocoa husks were then used to test the pelleting process. These tests were done on a KAHL33-390 pellet press. The pellet tests were conducted to determine if this technology can be used under standard operating conditions (technology parameters, tool shape) in order to produce pellets of the highest quality. Figure 14 Figure 15 depict the pellets produced. The used pelletizing press was designed primarily to pellet wood biomass. The pellet diameter was $6 \mathrm{~mm}$. The pelleting tests using the cocoa husks from both coarse and fine fractions went without major technical or technological problems. The process was actually very smooth. The physical-mechanical indicators of pellet-quality were average to lower than expected. This can be attributed to the chemical composition of the pelletized material (lignin amount, hemicelluloses amount, etc.). To determine mechanical quality indicators as well as chemical-thermal quality indicators, the pellets were subject to laboratory testing. Table 2 summarizes the results.

\section{CONCLUSIONS AND RECOMMENDATIONS}

The results show that cocoa husks can be pelleted using pelleting methods with satisfactory production quality. Refining the fraction (crushing) was not necessary. It was not possible to confirm the expected effect of finer fractions on pellet quality. Both fraction sizes had approximately equal values of the basic mechanical indicators that indicate pellet quality, which were bulk density, particle density and mechanical durability. The bulk density for pellets made with course fractions was 1.43 times greater than that of pellets made from crushed fractions. By pelleting, the bulk density of the cocoa husks supplied can be increased by 4.23fold. Because of the construction principle used and the impact of the size and shape of the pressing holes, the pelleting technology produced better quality extrusions that the briquetting technique. The pellet die was found to be the best solution for pressing cocoa husks. This results in better bonding and thus higher quality extrusions. From the monitored variables point of view the findings can be withdrawn as:

- With increasing of compression pressure also increases the extrusions density,

- Positive effect of particle size decreasing on extrusion density wasn't proven,

- Higher extrusions dilatation at extrusion from fine fraction was determined.

\section{ACKNOWLEDGEMENTS}

The paper is a part of the research done within the project APVV-19-0607 "Optimized progressive shapes and unconventional composite raw materials of high-grade biofuels" funded by the Slovak Research and Development Agency and 
the project VEGA 1/0085/19 „Research of acting forces interaction during densification of biomass and shape optimization of pressing tools of densification machines" funded by the Ministry of Education of Slovak Republic and to the Slovak Academy of Sciences.

\section{REFERENCES}

Arce, M.E. ; Saavedra, A. ; Míguez, J.L. ; Granada, E. ; Cacabelos, A. (2013) biomass fuel and combustion conditions selection in a fixed bed combustor. Energies, 6, 5973-5989. Retrieved from https://doi.org/10.3390/en6115973

Fernandes U., Costa M., (2012) Particle emissions from a domestic pellets-fired boiler, Fuel Processing Technology, 103, 51-56, Retrieved from https://doi.org/10.1016/j.fuproc.2011.08.020

Garcia Maraver, A., Zamorano, M. ; Fernandes, U. ; Rabacal, M. and Costa, M. (2014) : Relationship between fuel quality and gaseous and particulate matter emissions in a domestic pellet-fired boiler, Fuel, 119, 141-152, Retrieved from https://doi.org/10.1016/j.fuel.2013.11.037

Kaliyan, N., Morey Vance, R. (2009) : Factors affecting strength and durability of densified biomass products, Biomass and Bioenergy, 33 (3), 337-359, Retrieved from https://doi.org/10.1016/j.biombioe.2008.08.005

Kracík, P. ; Baláš, M. ; Lisý, M. ; Pospíšil, J. (2016) The size effect of heat-transfer surfaces on boiling. Materials and technology, 50, 939-944. Retrieved from https://doi.org/10.17222/mit.2015.245

Križan, P. (2021) Biomass Compaction. The Effects of Pressing Chamber Design Parameters on Extrusion Quality. 1st ed., Springer Nature, Switzerland,, p.162. Retrieved from https://doi.org/10.1007/978-3-030-89956-1_1

Križan, P. (2015) The densification process of wood waste, 1st ed. ; De Gruyter Open : Berlin, Germany, ; p. 184. Retrieved from https://doi.org/10.2478/9783110440027

Križan, P., Šooš, L., Matúš, M., Beniak, J., Svátek, M. (2015) : Research of significant densification parameters influence on final briquettes quality, Wood research, 60, 2, 301-315. Retrieved from https://www.researchgate.net/profile/Peter-Krizan-

2/publication/274316802_Research_of_significant_densification_paramete rs_influence_on_final_briquettes_quality/links/551bb2b20cf251c35b50a1 e8/Research-of-significant-densification-parameters-influence-on-finalbriquettes-quality.pdf

Križan, P. ; Matúš, M. ; Šooš, L'. ; Beniak, J. (2015) Behaviour of beech sawdust during densification into a solid biofuel, Energies, 8, 6382-6398 Retrieved from https://doi.org/10.3390/en8076382

Nielsen, N.P.K., Gardner, D.J. and Poulsen, T. (2009) : Importance of temperature, moisture content, and species for the conversion process of wood residues into fuel pellets, Wood and Fiber Science, 41(4), 414-415, Retrieved from https://wfs.swst.org/index.php/wfs/article/view/469

Standard DIN 51731 :(1996), Testing of solid fuels - compressed untreated wood, requirements and testing. Berlin, Germany : Deutsches Institut für Normung

Standard DIN 52182 :(1976) Bestimmung der Rohdichte. Berlin, Germany : Deutsches Institut für Normung. 
Standard EN ISO 17225-2 :(2014), Solid biofuels - Fuel specifications and classes Part 2 : Graded wood pellets ; European Committee for Standardization, Brussels, Belgium, 2014.

Standard EN ISO 17225-6 :(2014), Solid biofuels - Fuel specifications and classes Part 6 : Graded non-woody pellets, European Committee for Standardization, Brussels, Belgium, 2014.

Standard EN ISO 17828 :(2015), Solid biofuels - Determination of bulk density European Committee for Standardization, Brussels, Belgium, 2015.

Standard EN ISO 17829 :(2016), Solid Biofuels-Determination of length and diameter of pellets. European Committee for Standardization : Brussels, Belgium, 2016.

Standard EN ISO 18134-3 :(2016), Solid Biofuels-Determination of moisture content. Oven dry method. Part 3 : Moisture in general analysis sample ; European Committee for Standardization: Brussels, Belgium, 2016 